Artículo producto de la investigación

\title{
Determinación de metabolitos secundarios a partir de Bacillus subtilis con efecto biocontrolador sobre Fusarium sp.
}

\author{
Ariza Yesid ${ }^{1}$, Sánchez Ligia MSc. ${ }^{2}$ \\ ${ }^{1}$ Profesional Joven Investigador. Universidad Colegio Mayor de Cundinamarca, Bogotá, D.C. Colombia \\ ${ }^{2}$ Docente Investigadora. Universidad Colegio Mayor de Cundinamarca, Bogotá, D.C. Colombia.
}

Recibido: 09/08/2012 / Aceptado: 14/09/2012

Correspondencia: simpsoni6@hotmail.com

\begin{abstract}
Resumen
El uso de agentes de control biológico es una alternativa eficaz para el tratamiento de las enfermedades en plantas, contribuyendo en la disminución del uso de pesticidas químicos. Microrganismos del género Bacillus se han convertido en el centro de interés por su producción de metabolitos de tipo secundario, con propiedades antifungicas, supresores efectivos contra diversos fitopatógenos como Fusarium, Pythium, Phytophthora y Rhizoctonia. El objetivo del presente estudio fue identificar la presencia de metabolitos secundarios obtenidos por fermentación en estado líquido a partir de una cepa de Bacillus subtilis, los cuales fueron analizados mediante cromatografía de alta resolución (HPLC). Se confirmó su efecto biocontrolador sobre Fusarium sp por pruebas de excavación en placa. Se identificó el antibiótico Iturina A, con una concentración aproximada de $151.805 \mathrm{mg} / \mathrm{l}$ y las pruebas de antagonismo in vitro expresaron un porcentaje de 70 a $100 \%$ de inhibición de crecimiento sobre Fusarium sp. Se espera a futuro identificar la presencia de otros metabolitos de tipo secundario, de importancia en fitosanidad, como la producción de surfactina y fengicina.
\end{abstract}

Palabras Clave: B. subtilis, Biocontrol, cromatografía de alta resolución (HPLC), Iturina A, Fusarium sp.

\section{Determination of secondary metabolites from Bacillus subtilis with effect biological control on Fusarium sp.}

\begin{abstract}
The use of microorganisms for biological control of plants diseases in an effective alternative that reduce pesticides use that affect soil fertility. Bacillus genus has become a point of interest due the production of secondary metabolites with antifungal properties effective against several phytopathogenics such as Fusarium, Pythium, Phytophthora and Rhizoctonia. The aim of this study was to identify the presence of secondary metabolites produced by liquid fermentation from Bacillus subtilis, analyzed by High-performance liquid chromatography (HPLC), and confirm its biocontrol effect on Fusarium sp. The antibiotic iturin A was identified, with a concentration around $151,805 \mathrm{mg} / \mathrm{l}$. In vitro antagonism tests showed 70 to $100 \%$ growth inhibition of Fusarium sp. In the near future is expected to identify the existence of other secondary metabolites with possible significant effect on the plant protection, such as surfactin and fengycin.

Key words: B. subtilis, Biocontrol, Fusarium sp, High-performance liquid chromatography (HPLC), Iturina A.
\end{abstract}




\section{Introducción}

El uso excesivo de pesticidas y fertilizantes químicos en la agricultura moderna ha dado lugar al deterioro en la fertilidad de los suelos y a la aparición de formas de resistencia de los microrganismos fitopatógenos en todo el mundo (1). Para hacer frente a estos problemas, productos de origen microbiano y fertilizantes orgánicos son una alternativa limpia en el control fitosanitario.

Un agente de biocontrol es el Bacillus subtilis, bacteria Gram positiva que produce una gran cantidad de lipopeptidos, metabolitos primarios o secundarios, con amplio espectro antibiótico. Dichos metabolitos son supresores efectivos de algunos patógenos de plantas incluyendo; especies de Fusarium, Pythium, Phytophthora, Rhizoctonia, Sclerotinia, Septoria, y Verticillium (2-3).

Dentro de los compuestos producidos por Bacillus sp., se incluyen la surfactina, fengicina, iturina $\mathrm{A}, \mathrm{B}, \mathrm{y} \mathrm{C}$, micosubtilinas, $\mathrm{y}$ bacilomicinas, biosurfactantes activos de membrana con potentes actividades antimicrobianas (4). La surfactina se identificó por primera vez como un inhibidor de la formación del coágulo de fibrina. Pero de igual forma exhibe funciones antimicrobianas, anti-tumorales y anti-virales (5), e inhibe la formación de biopelículas de otras bacterias al interferir con la unión de las células a las superficies (6). El gen que codifica la producción de este polipeptido es el srfA, que contiene cuatro marcos de lectura abiertos (ORFs), srfA-A, srfA-B/, srfA-C, y srfA-Te respectivamente (7).

La familia Iturina comprende la bacilomicina, iturina y micosubtilina los cuales son lipoheptapeptidos cíclicos unidos por un residuo $\beta$-amino acido. Los miembros de esta familia tienen una fuerte propiedad antibiótica y una moderada actividad surfactante (8-10). El grupo de genes de bacilomicina $\mathrm{D}$ (bam/bmy), micosubtilina (myc), y iturina A (itu) tiene cuatro ORFs. (11,12), y codifican enzimas multifuncionales de acidos grasos sintasa, aminotranferasa y péptidos sintetasa (13-14).
La fengicina es un antifungico que inhibe hongos filamentosos pero es inefectivo contra levaduras y bacterias, contiene cinco subunidades no ribosomales de péptidos sintetasas (NRPS): FenC (287 $\mathrm{kDa})$, FenD (290 kDa), FenE (286 kDa), FenA (406 kDa), y FenB (146 kDa) (14). A continuación se presentan las estructuras primarias de algunos de los lipopeptidos producidos por cepas de Bacillus spp.

Surfactina: FA- $\beta$-OH-L-Glu-L-Leu-D-Leu-L-ValL-Asp-D-Leu-L-Leu

Fengicina: FA- $\beta$-OH -L-Glu-D-Orn-D-Tyr-D$a$ Thr-L-Glu-D-[A6]-L-Pro-L-Gln-L-Tyr-L-Ile $\mathrm{A} 6=\mathrm{Ala} / \mathrm{Val}$

Iturina A: FA- $\beta$-NH2-L-Asn-D-Tyr-D-Asn-LGln-L-Pro-D-Asn-L-Ser

Iturina C: FA- $\beta$-NH2-L-Asp-D-Tyr-D-Asn-LGln-L-Pro-D-Asn-L-Ser

Estos lipopéptidos, metabolitos de tipo secundario representan una importante herramienta en el desarrollo de nuevos productos eficaces contra patógenos de plantas de interés mundial, como es el caso de Fusarium sp causante de la enfermedad conocida como pudrición seca, marchitez, fusariosis o secadera, la cual ocasiona una muerte prematura de la planta por marchitamiento, al que también se asocia la obstrucción de haces vasculares, clorosis foliar leve, posterior defoliación y pudrición a nivel radical (15). En Colombia se presentan daños por Fusarium sp. principalmente en el Valle del Cauca, Meta y zona bananera del Magdalena (16).

El objetivo de este estudio fue determinar la presencia de metabolitos secundarios a partir de una cepa de B. subtilis, diseñando un proceso de fermentación liquida y recuperación de los mismos por procesos físicos y químicos, analizados posteriormente por cromatografía liquida de alta resolución (HPLC) y espectrometría de masas, realizando pruebas de antagonismo in vitro contra Fusarium sp. 


\section{Materiales y métodos}

Cepa bacteriana. La cepa UCMC TB2 correspondiente a $B$. subtilis, fue suministrada por la Colección de Microorganismos de la Universidad Colegio Mayor de Cundinamarca conservada a $-70^{\circ} \mathrm{C}$, obtenida originariamente de la rizosfera de Ornithogalum umbellatum (estrella de Belén). El microorganismo se recuperó en caldo infusión cerebro corazón (BHI) de Sharlau, se incubó a $37^{\circ} \mathrm{C}$ durante 24 horas y posteriormente se realizó un aislamiento en agar sangre, según protocolo descrito por Corrales L, Sánchez L (17).

Caracterización fenotípica de la cepa UCMC TB2. Se realizó coloración de Gram para verificar identidad y se confirmó su comportamiento bioquímico mediante la prueba de identificación bacteriana BBL- CRYSTAL, de acuerdo con las indicaciones de la casa comercial Becton Dickinson and Company.

Preparación de medios de cultivo. Para el precultivo del microrganismo se utilizó el medio modificado de Luria-Bertani (L), que contiene $10 \mathrm{~g}$ de polypeptona, $5 \mathrm{~g}$ de extracto de levadura y $5 \mathrm{~g}$ de $\mathrm{Nacl}$ por litro y se ajustó a $\mathrm{pH}$ 7.0. Posteriormente para el proceso de fermentación se utilizó el medio modificado $3 \mathrm{~s}$ con $30 \mathrm{~g}$ de polypeptona, $10 \mathrm{~g}$ de glucosa, $1 \mathrm{~g}$ de $\mathrm{KH} 2 \mathrm{PO} 4$ y $0.5 \mathrm{~g}$ de Mg SO $47 \mathrm{H} 20$ por litro a $\mathrm{pH} 6.8$.

Fermentación liquida. Un stock de B. subtilis fue inoculado en $100 \mathrm{ml}$ de medio LB e incubado durante 18 horas a $37^{\circ} \mathrm{C}$ y $180 \mathrm{rpm}$. Posteriormente $1 \mathrm{ml}$ del precultivo fue inoculado en $100 \mathrm{ml}$ del medio 3 s, dejándolo en agitación constante durante 24 horas, 37 C y $150 \mathrm{rpm}$.

Extracción de metabolitos. Para la extracción de los metabolitos secundarios, fueron adicionados 45 $\mathrm{ml}$ de metanol al producto obtenido por fermentación en Shaker, esta mezcla fue dejada en agitación constante durante una hora a $150 \mathrm{rpm}$. El extracto de metanol obtenido fue centrifugado a $5000 \mathrm{rpm}$ durante $10 \mathrm{~min} \mathrm{a} 4^{\circ} \mathrm{C}$, el sobrenadante se filtró utilizando una membrana de nitrocelulosa con tamaño de poro $0.45 \mu \mathrm{m}$. Se recolectó el filtrado para utilizarlo posteriormente en el proceso de identificación por cromatografía de alta resolución (HPLC) de acuerdo al protocolo descrito de S. Mizumoto, M. Hirai (1).

Identificación de metabolitos secundarios por cromatografía en fase reversa. El análisis cromatográfico se llevó a cabo en un cromatógrafo líquido ultrarrápido Shimadzu Prominence (Maryland, CA, EUA), acoplado a un detector selectivo de masas LCMS-2020. Los análisis se realizaron en una columna C18 Shim Pack con $75 \mathrm{~mm}$ de longitud y $2.0 \mathrm{~mm}$ de diámetro. El volumen de inyección de la muestra fue de $1 \mu \mathrm{l}$ y la fase móvil empleada correpondió a acetonitrilo con ácido fórmico al $0,1 \%(\mathrm{P} / \mathrm{V})$ y acetato de amonio $5 \mathrm{mM}$ en agua Milli-Q. Para la identificación de los metabolitos se usó un estándar externo de Iturina A obtenido de la casa comercial Sigma Aldrich. El control del sistema cromatográfico y la adquisición y el procesamiento de datos se realizó con el software Lab Solutions versión 3.5.

Determinación de la actividad biocontroladora de los metabolitos secundarios contra fusarium sp.

Recuperación del fitopatogeno Fusarium sp. El Fusarium sp., fue provisto por la Colección de Microorganismos de la Universidad Colegio Mayor de Cundinamarca. El hongo fue sembrado en agar papa dextrosa (PDA) e incubado a $25^{\circ} \mathrm{C}$ durante 7 días.

Control biológico in vitro. Para realizar las pruebas de antagonismo in vitro se utilizó el método de excavación en placa según Mitidieri, 1998 (18), con algunas modificaciones; en una caja con PDA se realizaron cinco perforaciones de $5 \mathrm{~mm}$, una en el centro de la caja y las restantes en las orillas con disposición equidistante, se procedió a colocar con micropipeta, $100 \mu \mathrm{l}$ del metabolito activo extraído y se colocó micelio del hongo fitopatógeno con 7 días de crecimiento en 4 puntos de la caja ubicados 
a una misma distancia, se incubó a $25^{\circ} \mathrm{C}$ durante 7 días. Se realizó el mismo procedimiento para el control negativo de la prueba con agua destilada estéril. Finalmente, se determinó el porcentaje de inhibición producido por el extracto de metabolitos activos de las cepa de $B$. subtilis utilizando la siguiente formula: (R1 - R2)/R1 x 100 según Whipps 1987 (19), donde R1 corresponde a la distancia de crecimiento del hongo en dirección a la bacteria antagonista y R2 la distancia entre el hongo y la bacteria (zona de inhibición).

Aislamiento y caracterización fenotípica del bacilo. El aislamiento en agar sangre permitió observar las características macroscópicas propias de B. subtilis, colonias mucoides con bordes ondulados y extendidos en el medio. En la coloración de Gram se observaron bacilos Gram positivos con bordes redondeados y presencia de esporas, figura 1.

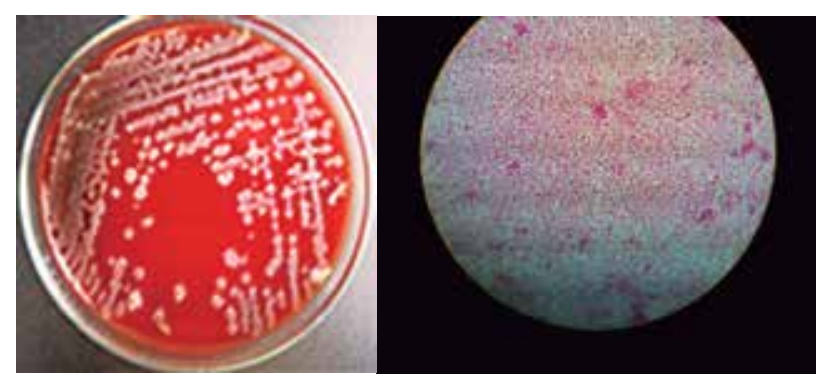

Figura 1. Cultivo y coloración de Gram de B. subtilis.

Los resultados obtenidos por BBL- CRYSTAL indicaron un 98.1\% de homología para Bacillus subtilis.
Identificación de metabolitos secundarios. El análisis por cromatografía líquida de alta resolución mostró la presencia de Iturina A, un polipéptido con fuertes propiedades antifúngicas, el tiempo de retención del metabolito encontrado en la muestra fue a los 2, 31 min presente con una concentración aproximada de $151.805 \mathrm{mg} / \mathrm{l}$, figura 2 . Los espectros de masas obtenidos para el estándar y para la muestra mostraron una concordancia correspondiente al 98\%, dato que confirmo la presencia de Iturina A en la muestra analizada, figura 3.

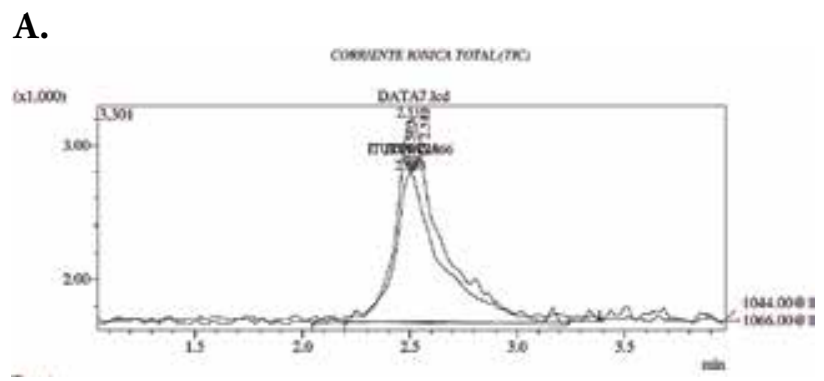

B.

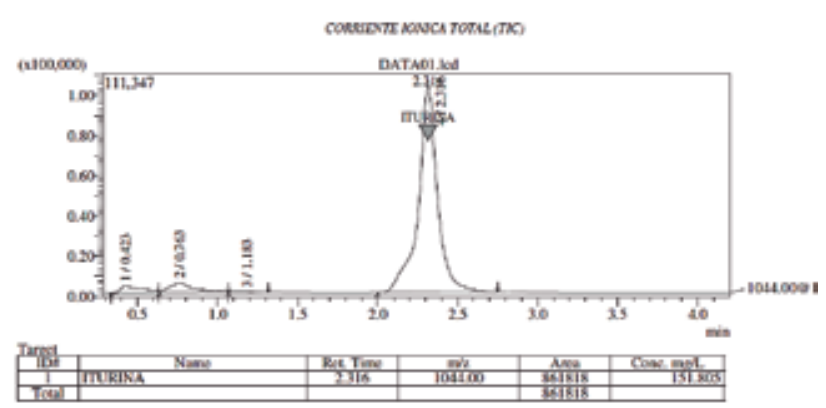

Figura 2. Cromatograma correspondiente al estándar (A) y la muestra (B).

A.

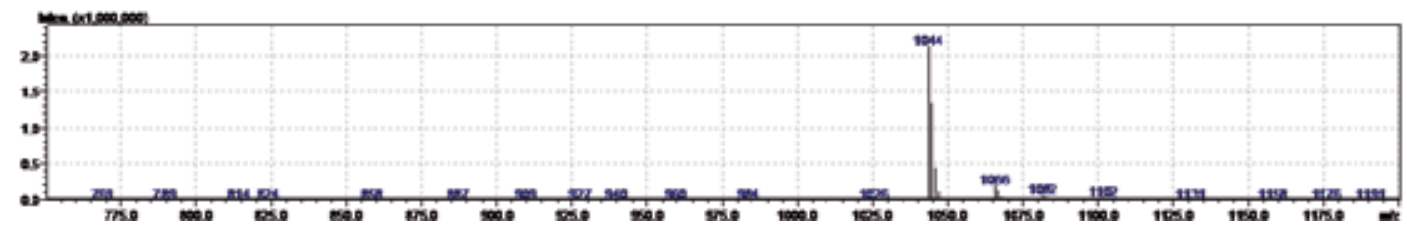

B.

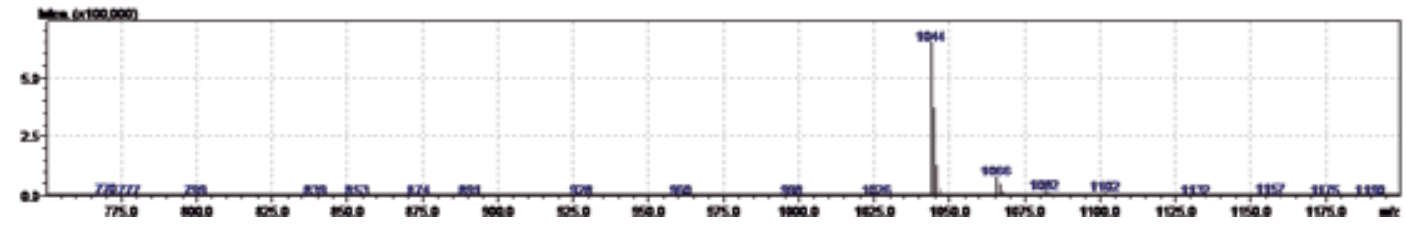

Figura 3. Espectro de masas del estándar (A) y de la muestra (B). 
Control Biológico in vitro. El extracto del metabolito activo de $B$. subtilis resultó ser eficiente contra el ataque de Fusarium sp en la prueba in vitro. Los porcentajes de inhibición fueron positivos entre el 70 y $100 \%$ en las 5 repeticiones realizadas, como se muestra en la tabla 1 y figura 4 .

Tabla 1. Porcentaje de inhibición de crecimiento del Fusarium $s p$., con respecto a las cinco repeticiones de extracto del metabolito activo de B. subtilis.

\begin{tabular}{|cc|}
\hline Repetición & $\begin{array}{c}\text { \% Inhibición } \\
\text { de crecimiento }\end{array}$ \\
\hline 1 & $86.66 \%$ \\
\hline 2 & $80 \%$ \\
\hline 3 & $93.3 \%$ \\
\hline 4 & $76.6 \%$ \\
\hline 5 & $83.3 \%$ \\
\hline
\end{tabular}
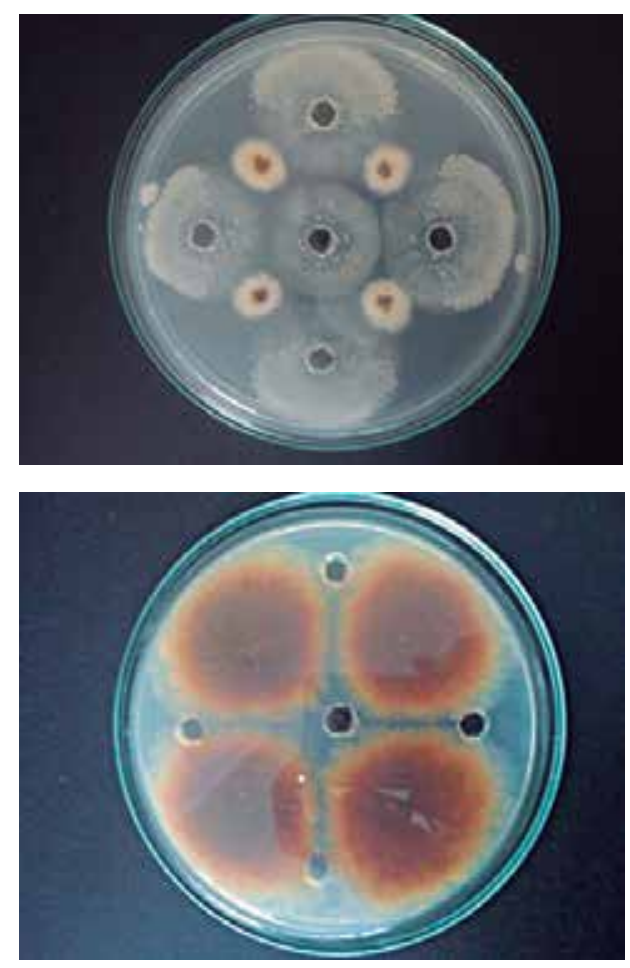

Figura 4. Pruebas de antagonismo in vitro. Cada pozo representa una repetición, la primera corresponde a la ubicada en la parte superior y el resto se disponen de acuerdo a las manecillas del reloj.

Recientemente agentes de control biológico para el tratamiento de las enfermedades en plantas han recibido una considerable atención, con el propósito de reducir el uso de químicos en la agricultura
(20). Bacterias Gram positivas como B. subtilis, han demostrado ser un excelente agente de tipo biológico, son productores de una amplia gama de péptidos, metabolitos de tipo secundario con actividad antifúngica.

Los biosurfactantes son compuestos biológicamente activos de superficie producidas por una amplia variedad de microorganismos. Tienen excelentes propiedades como la baja toxicidad y alta biodegradabilidad, son capaces de disminuir la tensión superficial y por lo tanto son posibles sustitutos para agentes tensioactivos. Por lo tanto, son prometedores para su uso en la agricultura, industria alimentaria, farmacéutica, cosmética, industrias de producción de petróleo, y procesos de biorremediación. La porción hidrófoba de estas moléculas es de ácidos grasos (saturados, insaturados o hidroxilados), mientras que la parte hidrófila se compone generalmente de péptidos, mono-, di-, o polisacáridos (21). La producción de lipopéptidos con capacidad biosurfactante (LPBSs) fue reportada por primera vez a partir de Bacillus subtilis (22). Desde entonces, varios tipos de LPBSs con actividad superficial significativa y anti-microbiana se han aislado de cepas de Bacillus. La organización sistemática de los péptidos sintetasa no ribosomales (NRPSs) permite la alteración estructural de lipopéptidos mediante el cambio de dominios para crear nuevos lipopéptidos (23).

La mayoría de LPBSs de Bacillus se pueden clasificar en tres familias: surfactina, fengicina y iturina. Hay varias cepas de Bacillus que tienen la capacidad de producir las tres familias de LPBSs al mismo tiempo (24). Además de estas tres familias, otros lipopéptidos han sido identificados en las especies de Bacillus.

En este estudio se determinó la presencia de Iturina A, un polipéptido cíclico que contiene 7 residuos de $\alpha$-aminoacidos (L-Asn-D-Tyr-D-Asn-L-Gln-LPro-D-Asn-L-Ser-) y un residuo de $\beta$-aminoacido. Su producción se realizó por fermentación en estado líquido y se obtuvo durante la fase estacionaria 
de crecimiento. El medio 3s utilizado favoreció el crecimiento del microrganismo, por su alto contenido de polipeptona como fuente de nitrógeno y glucosa como fuente de carbono, demostrando que la biosíntesis de antibióticos esta directamente influenciada por los diferentes factores nutricionales y ambientales (25).

Se ha informado que la producción de la Iturina y fengicina es inducida por inanición y regulada por los productos de los genes spo0A asociados con la esporulación, esta relación se atribuye a la etapa del cultivo y el orden de producción, aunque se lleven a cabo en diferente fases de crecimiento, al final de la etapa de esporulación, se termina la producción de los lipopeptidos (26).

B. subtilis se considera que es un aerobio estricto, pero varios grupos han demostrado que esta especie bacteriana es capaz de crecer incluso con un suministro de oxígeno limitado. Se encontró que el suministro suficiente de oxígeno disuelto (DO) desempeña un papel crucial en la eficiente producción de lipopéptidos (27).

La extracción y purificación de la Iturina A se realizó con metanol por su alto grado de afinidad y por procesos físicos de centrifugación y filtración, permitiendo obtener un extracto clarificado libre de partículas y restos celulares, que garantizaron los buenos resultados en el proceso de identificación por HPLC y espectrometría de masas.

Las pruebas de antagonismo in vitro contra Fusarium sp presentaron unos resultados positivos con un 70 a $100 \%$ de inhibición de crecimiento, demostrando la gran capacidad de la Iturina A como un agente de control biólogo frente a fitopatogenos de interés mundial. Su importancia se puede conferir a su forma de interactuar con las moléculas de colesterol, interrumpiendo la membrana citoplasmática del hongo, creando canales transmembranales, que permiten la liberación de iones vitales como el $\mathrm{K}+(28)$.
De acuerdo con los resultados del presente estudio, se puede sugerir el uso de genes de amplificación para mejorar la producción y actividad de la Iturina A y posteriormente realizar la formulación de un producto activo que pueda remplazar el uso de algunos productos químicos. Aunque el éxito comercial de los tensioactivos microbianos se encuentra limitado por su alto costo de producción. La optimización de crecimiento y producción con sustratos más económicos, renovables, nuevos y eficientes métodos de procesamiento, podría hacer que la producción de lipopeptidos sea más rentable y viable económicamente. Además, cepas microbianas recombinantes, capaces de crecer en una amplia gama de sustratos, permitiría producir metabolitos secundarios con alto rendimiento.

Agradecemos a Colciencias y la Universidad Colegio Mayor de Cundinamarca por su apoyo a la formación de jóvenes investigadores y correspondiente financiación del proyecto.

\section{Referencias}

1. S. Mizumoto . M. Hirai . M. Shoda. Production of lipopeptide antibiotic iturin A using soybean curd residue cultivated with Bacillus subtilis in solid-state fermentation. Appl Microbiol Biotechnol. 2006; 72: 869-875.

2. Naorska, K., Bikowski, M. Multicellular behaviour and production of a wide variety of toxic substances support usage of Bacillus subtilis as a powerful biocontrol agent. Acta Biochimica Polonica. 2007; 54: 495-508.

3. Bais, H.P., Fall, R., Vivanco, J.M., Biocontrol of Bacillus subtilis against infection of Arabidopsis roots by Pseudomonas syringae is facilitated by biofilm formation and surfactin production. Plant Physiology. 2004; 134: 307-319.

4. Muaaz Mutaz Al-Ajlani, Muhammad Abid Sheikh, Zeeshan Ahmad and Shahida Hasnain. Production of surfactin from Bacillus subtilis MZ-7 grown on pharmamedia commercial medium. Microbial Cell Factories. 2007; 6:17.

5. Rodrigues, L.; Banat, I.M.; Teixeira, J.; Oliveira, R. Biosurfactants: potential applications in medicine. J. Antimicrob. Chemother. 2006; 57: 609-618.

6. Mireles, J.R.; Toguchi, A.; Harshey, R.M. Salmonella enterica serovar typhimurium swarming mutants with altered biofilmforming abilities: surfactin inhibits biofilm formation. J. Bacteriol. 2001; 183: 5848-5854.

7. Niran Roongsawang, Kenji Washio and Masaaki Morikawa. Review Diversity of Nonribosomal Peptide Synthetases Involved in the Biosynthesis of Lipopeptide Biosurfactants, International Journal of Molecular Sciences ISSN 1422-0067, Int. J. Mol. Sci. 2011; 12: 141-172. 
8. Roongsawang, N.; Thaniyavarn, J.; Thaniyavarn, S.; Kameyama, T.; Haruki, M.; Imanaka, T.; Morikawa, M.; Kanaya, S. Isolation and characterization of a halotolerant Bacillus subtilis BBK-1 which produces three kinds of lipopeptides: bacillomycin L, plipastatin, and surfactin. Extremophiles. 2002; 6: 499-506.

9. Isogai, I.; Takayama, S.; Murakoshi, S.; Suzuki, A. Structure of $\beta$-amino acids in antibiotics iturin A. Tetrahedron Lett. 1982, 23: 3065-3068.

10. Leclere, V.; Marti, R.; Bechet, M.; Fickers, P.; Jacques, P. The lipopeptides mycosubtilin and surfactin enhance spreading of Bacillus subtilis strains by their surface-active properties. Arch. Microbiol. 2006; 186: 475-483.

11. Moyne, A.L.; Cleveland, T.E.; Tuzun, S. Molecular characterization and analysis of the operon encoding the antifungal lipopeptide bacillomycin D. FEMS Microbiol. Lett. 2004; 234: 43-49.

12. Tsuge, K.; Akiyama, T.; Shoda, M. Cloning, sequencing, and characterization of the iturin A operon. J. Bacteriol. 2001, $183,6265-6273$.

13. Hansen, D.B.; Bumpus, S.B.; Aron, Z.D.; Kelleher, N.L.; Walsh, C.T. The loading module of mycosubtilin: an adenylation domain with fatty acid selectivity. J. Am. Chem. Soc. 2007; 129: 6366-6367.

14. Wu, C.Y.; Chen, C.L.; Lee, Y.H.; Cheng, Y.C.; Wu, Y.C.; Shu, H.Y.; Gotz, F.; Liu, S.T. Nonribosomal synthesis of fengycin on an enzyme complex formed by fengycin synthetases. J. Biol. Chem. 2007, 282: 5608-5616.

15. González, M., I. Torres y H. Guzmán. 2002. Búsqueda de resistencia natural contra patógenos de raíz Phytophthora capsici, Fusarium solani y Fusarium oxysporum en colectas de Chile. Proceedings of the 16th Internacional Pepper Conference. Tampico y Tamaulipas, México.

16. Juan Guillermo Cubillos Hinojosa1; Alberto Páez Redondo y Lauris Mejía Doria. Evaluation of the Biocontrol Capacity of Trichoderma harzianum Rifai against Fusarium solani (Mart.) Sacc. Associated to the Complex "Dryer" in Passion Fruit Under Greenhouse Conditions. Int. J. Mol. Sci. 2010; 11: 4526-4538.

17. Sánchez L, Corrales L, Lancheros A. Evaluación in vivo del potencial biocontrolador de morfotipos bacterianos nativos contra fusarium oxysporum y caracterización por PCR de los microorganismos más eficientes. Grupo Ceparium UCMC. 2008. Literatura gris.

18. Mitidieri, L. Control Biológico de hongos del suelo con Trichoderma. IDIA. 1988; 44:45-49.

19. Whipps, JM. 1987. Effect of media of growth and interactions between a range of soil-borne glass-house pathogens and antagonistic fungi. New Phytol.,107: 127-142.

20. S. Mizumoto \& M. Hirai \& M. Shoda. Enhanced iturin A production by Bacillus subtilis and its effect on suppression of the plant pathogen Rhizoctonia solani. Appl Microbiol Biotechnol (2007) 75:1267-1274.

21. Georgiou, G.; Lin, S.C.; Sharma, M.M. Surface-active compounds from microorganisms. Biotechnology (NY) 1992, 10: 60-65.

22. Arima, K.; Kakinuma, A.; Tamura, G. Surfactin, a crystalline peptidelipid surfactant produced by Bacillus subtilis: isolation, characterization and its inhibition of fibrin clot formation. Biochem. Biophys. Res. Commun. 1968; 31: 488-494.

23. Eppelmann, K.; Stachelhaus, T.; Marahiel, M.A. Exploitation of the selectivity-conferring code of nonribosomal peptide synthetases for the rational design of novel peptide antibiotics. Biochemistry 2002, 41: 9718-9726.

24. Kim, P.I.; Ryu, J.; Kim, Y.H.; Chi, Y.T. Production of biosurfactant lipopeptides iturin A, fengycin and surfactin A from Bacillus subtilis CMB32 for control of Colletotrichum gloeosporioides. J. Microbiol. Biotechnol. 2010; 20: 138-145.

25. Maget-Dana R, Peypoux F. Iturins, a special class of poreforming lipopeptides: biological and physicochemical properties. Toxicology. 1994;87: 151-174.

26. Mohammad Shahedur Rahman*, Takashi Ano, Makoto Sho$\mathrm{da}$, Second stage production of iturin A by induced germination of Bacillus subtilis RB14, Journal of Biotechnology. 2006; 125:513-515.

27. François Coutte \& Didier Lecouturier, Production of surfactin and fengycin by Bacillus subtilis in a bubbleless membrane bioreactor, Appl Microbiol Biotechnol. 2010; 87:499-507.

28. Jing Li, Qian Yang, Li-hua Zhao, Shu-mei Zhang, Yu-xia Wang, and Xiao-yu Zhao. Purification and characterization of a novel antifungal protein from Bacillus subtilis strain B29. J Zhejiang Univ Sci B. 2009; 10(4): 264-272. 